\section{HYPEREXTENSION AND HAEMATOMYELIA}

BY

P. N. ROBSON, M.B., F.R.C.S.

Senior Orthopaedic Registrar, Royal Victoria Infirmary, Newcastle-upon-Tyne

Spinal cord injury occurring without radiological evidence of the corresponding bone injury constitutes a well-recognized picture. The majority of cases are diagnosed as haematomyelia and are seen in medical clinics with a partial or complete lesion of the cervical spinal cord and no radiological evidence of bone injury or dislocation. This paper attempts to explain the actual mechanism of cord injury in these patients.

Jefferson (1928) stated that "in hyperextension the articular processes of the cervical vertebrae lock together to form a solid column of bone which is resistant to injury, and therefore hyperextension injuries fall chiefly on the atlas and axis." Much work has been done on these latter injuries, but hyperextension injuries of the rest of the cervical spine are less generally recognized.

Walshe (1944) gave an excellent description of the clinical features of haematomyelia and stressed its association with closed cerebral injury, without any explanation of the actual mechanism. He also emphasized the frequency of the Babinski inversion of the supinator jerk in such cases as indicating a focal lesion of the cervical cord at the level of the fifth segment.

Nattrass (1944) described the case of a soldier who on D Day dived from a landing craft into shallow water. He struck his head on the bottom and an immediate weakness of the legs and arms ensued. $X$-ray films of the neck being normal, the patient was labelled hysterical. On his admission to a neurological unit the diagnosis of haematomyelia was made. Nattrass stresses the danger of regarding these cases as functional.

Barnes (1948) first described the mechanism of fatal cervical cord compression without radiological evidence of fracture or dislocation. In his cases there had been a dislocation of the neck in hyperextension with spontaneous reduction. The post-mortem findings were rupture of the anterior common ligament at the level of the injury with disruption of the intervertebral disk, the dislocation recurring in forced extension. Taylor and Blackwood (1948) described a similar case.

Taylor (1951) described a case in which quadriplegia and death followed a hyperextension injury of the neck, radiography demonstrating no bone injury. At necropsy there was crushing of the cervical cord but no evidence of bone injury, dislocation, or disk prolapse. Crushing of the cord by the ligamentum flavum, which bulges forward prominently in hyperextension, was suggested as the causative mechanism.

\section{Mechanism of Cord Injury}

It is suggested that in the absence of radiological evidence of skeletal injury the crushing of the cervical cord may be due to either of two mechanisms: (1) massive central disk prolapse, or (2) hyperextension injuries.

\section{Massive Central Disk Prolapse}

This is much rarer than the common cervical disk syndrome associated with root irritation. It is due to a flexion injury, and it is doubtful whether it can occur in hyperextension. Cramer and McGowan (1944) published serial $x$-ray films of the cervical spine demonstrating this.

To elucidate this point discograms of excised specimens of the cervical spine have been made. Fig. 1 shows tracings of a discogram in a young subject. In the neutral position the normal outline of the nucleus pulposus is shown. In extension the nucleus moved forward as the posterior angles of the vertebral bodies approximated; thus posterior prolapse of the nucleus seems unlikely in hyperextension injuries.

\section{Hyperextension Injuries}

There are two methods of spinal cord injury which occur with forcible hyperextension of the neck.

Type 1.-This is a transient dislocation with tearing of the anterior common ligament and disruption of the
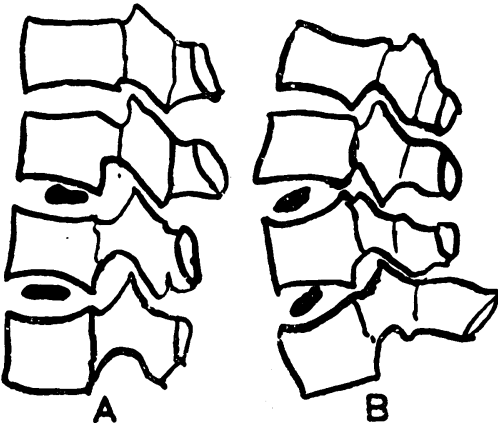

FIG. 1.-Tracings made from discograms of a normal cervical spine. A. In the line of the nucleus pulposus. B. In extension, showing the approximation of the posterior margins of the vertebral the posterior margin bodies and the squeezing forwards of the disks. neutral position, showing the normal out-

permitting separation of the vertebral bodies with crushing of the spinal cord. Fig. 2 is a post-mortem radiograph of the excised cervical spine from such a case, showing the mechanism of the injury. As soon as the hyperextending force is removed the dislocation is reduced by muscle pull exactly as in recurrent dislocation of the ankle due to rupture of the external ligament. In nonfatal cases repair of this lesion is by ossification of the torn anterior common ligament bridging the affected disk space. Fig. 3 shows two radiographs of a man who had a hyperextension injury of the neck. The first, taken immediately after the accident, shows avulsion of a flake of bone from the upper border of the sixth cervical vertebra by the torn anterior common ligament. The second film,

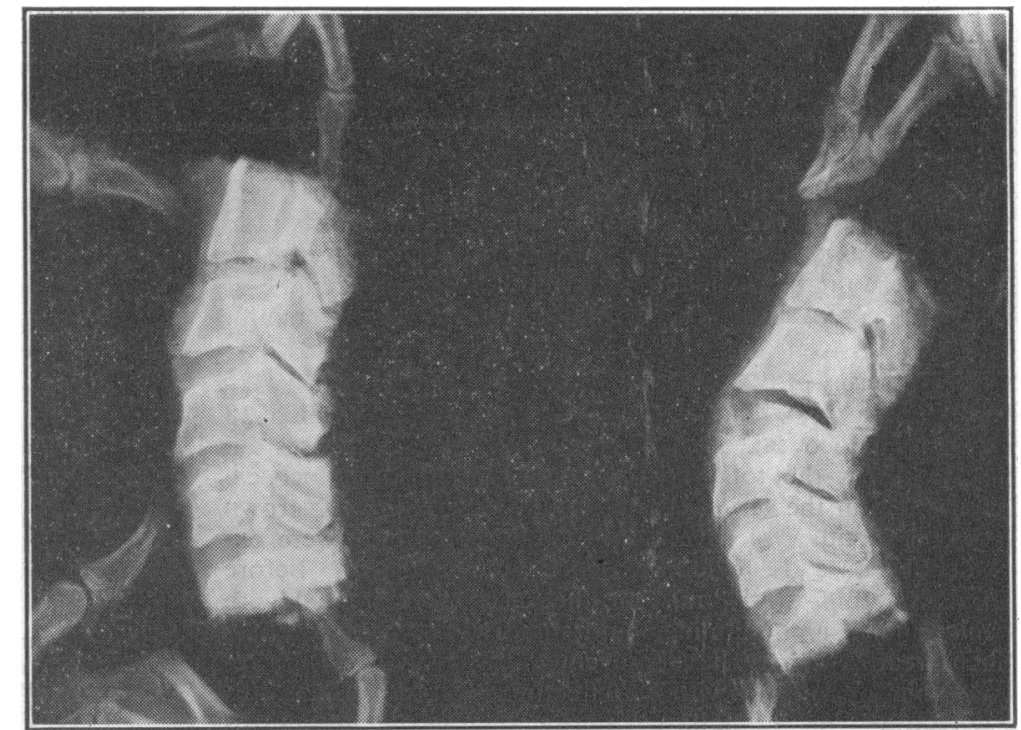

FIG. 2-Excised cervical spine from a fatal case of a type 1 hyperextension injury, in the neutral and hyperextended positions. 


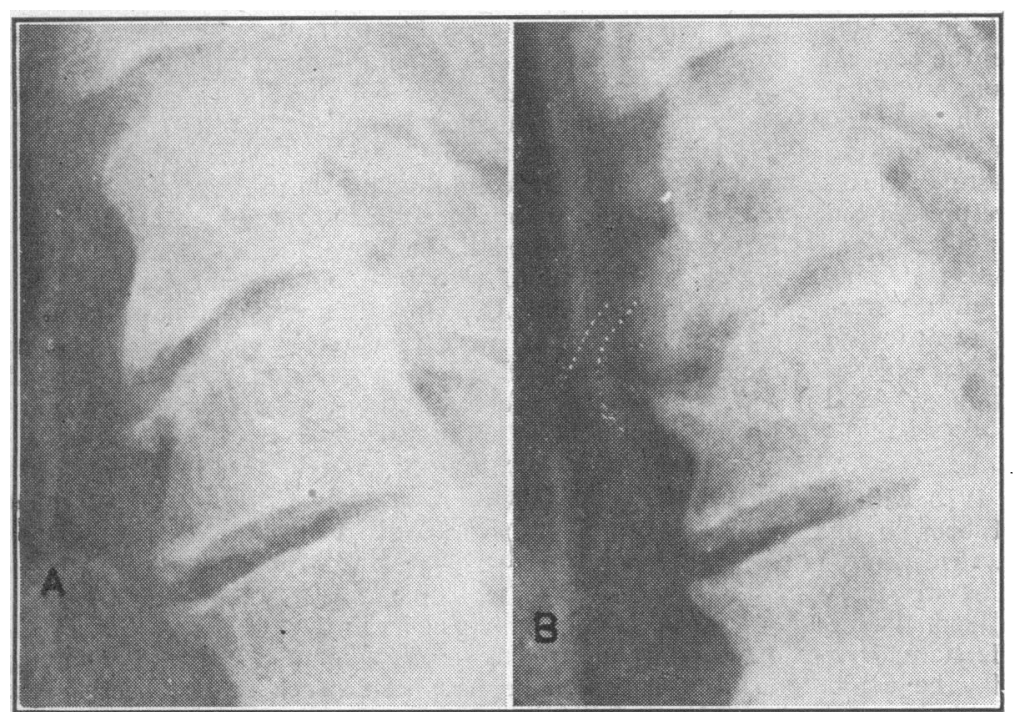

FIG. 3.- Lateral radiographs of a cervical spine following a hyperextension injury. A. Immediately after injury, showing avulsion of an osteophyte from the upper border of C 6. B, Eight months later, showing repair by ossification of the anterior common ligament.

taken eight months later, shows ossification of the damaged ligament bridging the intervertebral space. Fig. 4 is an outline drawing of Fig. 3.

Type 2.-In hyperextension of the normal cervical spine the ligamenta flava between the laminae bulge forward prominently. If there is pre-existing cervical spondylosis with ridging at the posterior aspects of the intervertebral disks, the cord may be crushed between the ligamenta flava and the posterior ridge. Fig. 5 shows two moulds of the cervical spinal canal in forced hyperextension. The first is a normal spine from a young adult, and the mould shows ridging posteriorly where the ligamenta flava have bulged forwards. The second mould is from an older body in which there was cervical spondylosis. In this case the mould is markedly narrowed between the ligamentum flavum behind and the ridging at the intervertebral spaces in front. Had forcible hyperextension of the patient's neck occurred during life she must have sustained a cervical cord lesion.

\section{Case Histories}

All cases diagnosed as haematomyelia in a neurological clinic from 1939 to 1954 inclusive were reviewed. Twelve cases were available.

Case 1.-A woman aged 35 was admitted in 1946 with a three-months history of gradual onset of numbness in the

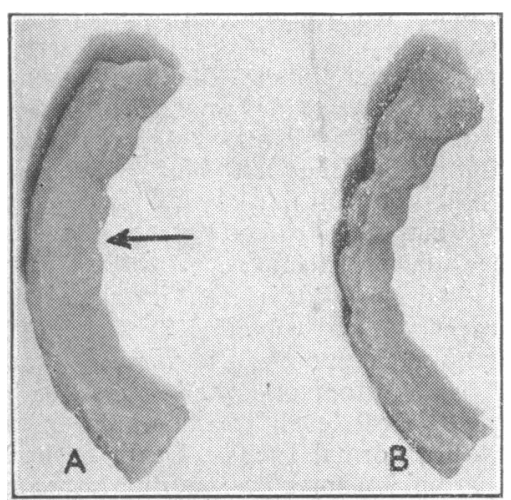

Fig. 5.-Photograph of moulds of the cervical spinal canal in hyperextension. A. From a normal cervical spine in a young man, showing ridging of the mould posteriorly (at arrow). B. from a woman aged 67. In this case there was spondylosis, and the mould is markedly narrowed. This is the mechanism of a type 2 hyperextension injury.
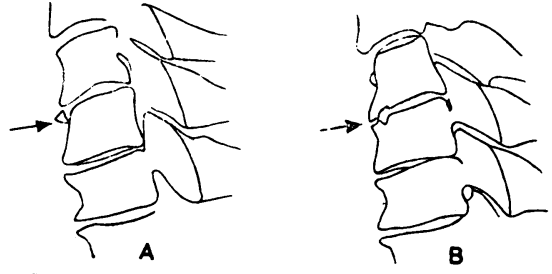

FIG. 4.-Tracing of Fig. 3. The arrows show: A, the avulsed osteophyte; B, the ossified ligament.

Cranial nerves, N.A.D. ; sensory blunting and loss of pain sense below the fifth cervical segment of the right side; the only muscle-wasting found was the left first dorsal interosseus; reflexes were normal except for the loss of the abdominal reflex on the left side. Radiographs of the cervical spine showed nothing abnormal.

Case 2.-A man aged 47 was admitted in 1940 following an accident in the black-out. After having drunk some pints of beer he tripped over a low fence and fell, striking his forehead on the ground. He was unable to rise owing to weakness of the legs. On admission he was found to have a spastic paraplegia with loss of pain sense on the left side of the trunk and in the left arm and leg. Atrophy of the small muscles of both hands rapidly followed. Radiographs of the cervical spine were stated to be within normal limits. He died of intercurrent disease eleven years after the injury.

Case 3.-A woman aged 54 was seen as an out-patient in November, 1939, with a history of having fallen downstairs some weeks previously, striking her forehead on a stair. She had noticed weakness of both hands. Examination confirmed the weakness of both hands, with hyperaesthesia. Present condition:-For several years after her injury she had considerable pain in her neck on movement. This cleared up spontaneously and she now has no symptoms. On examination the cervical spine had a full range of painless movement. C.N.S.: No abnormal physical signs were present. Radiographs of the cervical spine showed some neurocentral lipping and ossification of the anterior common ligament between the fifth and sixth cervical vertebrae.

Case 4.-Following an argument with a bus conductor in July, 1941, a man aged 61 received a blow in the face which jerked his head backwards. He was seen in the out-patient department some weeks later complaining of pain in the neck and difficulty in using the hands. On examination he was found to have astereognosis of the hands and gross painful limitation of neck movement. Radiographs of the cervical spine showed no evidence of fracture or dislocation. Present condition :- He complains of pain and clicking on neck movement. The difficulty in using the hands persists. On examination the cervical spine showed gross limitation of movement with severe pain on forcing. C.N.S.: No sensory loss or abnormality of reflexes was present. There was marked wasting of the small muscles of both hands and astereognosis persisted. Radiographs of the cervical spine demonstrated considerable spondylosis with some neurocentral lipping.

Case 5.-A man aged 73 fell forwards and struck his forehead on the ground in April, 1945. There was immediate weakness of both legs. He was seen in the out-patient department some weeks later complaining of weakness of both legs and difficulty in walking. On examination he was found to have a spastic paraplegia affecting both legs, with muscular weakness and wasting. There were no sensory changes found. Radiographs of the cervical spine showed slight posterior subluxation of the fourth on the fifth cervical vertebra with some spondylosis. An orthopaedic surgeon thought that these changes were part of a long-standing spondylosis. Present condition:- He com- 
plains of pain on neck movements and difficulty in walking owing to weakness of the legs. On examination the cervical spine had a full range of flexion, lateral flexion, and rotation. There was limitation of extension with pain on attempting this movement. C.N.S.:-Cranial nerves: N.A.D. Upper limbs: Marked interosseous wasting ; weakness of grip ; no sensory changes; reflexes were present but the right supinator jerk was inverted. Lower limbs: Wasting of all muscle groups with marked weakness; walking was only just possible; no sensory change; reflexes were present and equal and the plantar responses were flexor on both sides. Trunk : No sensory change ; abdominal reflexes were present. Radiographs of the cervical spine showed marked cervical spondylosis with retrospondylolisthesis between the fourth and fifth vertebrae.

Case 6.-A man aged 62 was admitted in 1950 with a history that after having had several pints of beer in his workmen's club he bent down to pick up a threepennypiece from the floor. He fell forward, striking his forehead on the floor. He was immediately paralysed, unable to move arms or legs, and unable to speak. He stated that his arms were flexed at the elbows. Next day he could again talk, and some power had returned to the limbs. On admission there was a paresis of arms and legs with loss of reflexes. During the ensuing four weeks, however, a partial recovery occurred, and at the time of his discharge from hospital, some five weeks after the accident, he was able to walk a few steps. Present condition:- $\mathrm{He}$ is barely able to walk with the aid of two sticks. On examination the cervical spine showed gross restriction of movement in all directions but no pain. C.N.S.:-Cranial nerves: N.A.D. Upper limbs: Wasting of all muscle groups in both arms, especially interossei; diminished power, with spasticity; reflexes increased; inversion of both supinator jerks; no sensory change. Lower limbs: Wasting of all groups, with weakness but only slight spasticity; reflexes increased; plantar responses-right extensor, left flexor. Trunk: Loss of abdominal reflexes on both sides. Radiographs of the cervical spine showed congenital fusion of the vertebral bodies of the second and third segments, also of the sixth and seventh with the first thoracic. There was ossification of the anterior common ligament between the fifth and sixth cervical vertebrae.

Case 7.-A man aged 54 was admitted after his motorcycle ran into a stationary car, causing facial lacerations and hyperextension of his neck. The lacerations were sutured and the following day he noticed weakness of both hands. On examination the cervical spine had a full range of flexion, lateral flexion, and rotation. Extension was limited and painful. C.N.S.:-Cranial nerves: N.A.D. Upper limbs: Wasting of interossei of both hands, with weakness of grip; sensory blunting in fourth and fifth fingers of the left hand; reflexes were present and equal, but the left supinator jerk was inverted. Lower limbs: N.A.D. Radiographs of the cervical spine showed a moderate degree of spondylosis, with fracture of an osteophyte at the upper anterior angle of the seventh cervical vertebra.

Case 8.-This patient, a woman aged 54, was seen in the out-patient department in 1941 four weeks after a fall downstairs. She was unable to give a clear history of the mechanism of the injury, and complained of pain in the neck and weakness of the left hand. On examination wasting of the muscles of the left hand and loss of thermal sensation were found. Present condition:-She has no symptoms referable to the accident. On examination the cervical spine showed slight limitation of the extremes of lateral flexion, otherwise there was full range of movement. C.N.S.: No alteration of sensation or muscle-wasting was found, but there was depression of the tendon reflexes of the left arm. Radiographs of the cervical spine showed moderate spondylosis and ossification of the anterior common ligament between the sixth and seventh vertebrae.

Case 9.-A woman aged 53 was admitted in 1943 after a fall downstairs in which she struck the back of her neck on a stair. She was unable to move after her accident. On examination complete paralysis of all four limbs was found. Present condition:-For the first three weeks after the accident she remained completely paralysed, but there had been a gradual recovery over the next three months, and since then her condition has been static. She is able to walk slowly with some difficulty. On examination the cervical spine showed limitation of the extremes of lateral flexion and rotation. No pain on movement. C.N.S.:Cranial nerves: N.A.D. Upper limbs: Wasting of the interossei of both hands, more pronounced on the right than on the left; no loss of sensation; tendon reflexes increased, with inversion of the right supinator jerk ; mild spasticity. Lower limbs: Slight spasticity; reflexes present and equal; plantar responses flexor. Trunk: No sensory change; loss of abdominal reflexes. Radiographs of the cervical spine showed gross spondylosis with ossification of the anterior common ligament between the fifth and sixth cervical vertebrae.

Case 10.-This patient, a man aged 49 , was seen in the out-patient department in 1947 , some weeks after a mining accident in which he had been struck on the back of the neck by a fall of stone. He was complaining of severe pain in the neck and weakness of the limbs. On examination he was found to have a partial spastic quadriplegia. Radiegraphs of the cervical spine showed no evidence of fracture or dislocation. Present condition:- - He complains of some pain in the neck on movement and of weakness of the legs and difficulty in walking. On examination the cervical spine showed limitation of extension and full range of other movements. C.N.S. :-Cranial nerves: N.A.D. Upper limbs: Slight spasticity; reflexes active and equal no muscle-wasting or sensory loss. Lower limbs: Marked spasticity, with wasting of all groups ; reflexes hyperactive plantar responses extensor. Trunk: No sensory change: abdominal reflexes absent. Radiographs of the cervical spine showed a moderate spondylosis, most marked in the fifth/sixth region.

Case 11.-A man aged 49 was seen in the out-patient department in 1949, six months after a mining accident in which he received a heavy blow on the back of his neck. He immediately noticed numbness of his left hand. He was complaining also of pain in the neck. On examination he was found to have marked limitation of cervical spine movement. Muscle-wasting was present in the left hand, with some sensory blunting. Radiographs of the cervical spine were not taken at this time. Present condition: He complains of severe pain in the neck for which he has to wear a plastic collar; the numbness and weakness of the left hand persists. On examination the cervical spine showed gross limitation of movement in all directions, with severe pain. C.N.S.:-Cranial nerves : N.A.D. Upper limbs : Weakness of grip of the left hand with wasting of the first dorsal interosseous muscle; sensory blunting over the whole of the left hand except the index finger; loss of tendon reflexes in the left arm. Lower limbs : Blunting of sensation to pin-prick in the left leg; no wasting or loss of power; loss of left ankle-jerk; plantar responses flexor. Trunk: Sensory blunting of the left half; abdominal reflexes present. Radiographs of the cervical spine show marked spondylosis between the sixth and seventh vertebrae, with sclerosis of the adjacent surfaces of the vertebral bodies.

Case 12.-A man aged 46 was admitted in June, 1951, after having been hit by a locomotive. There was a traumatic amputation of the left foot and a complete quadriplegia below the sixth cervical level. Radiographs of the cervical spine were within normal limits. Death occurred two weeks later, and permission for a post-mortem examination was refused.

\section{Review of Cases}

These 12 cases fall into three groups: non-traumatic ( 1 case), history of hyperextension injury (6 cases), and no clear history of mechanism (5 cases). 
Group 1.-This group contains only Case 1, and is outside the scope of this paper. Some other mechanism, such as an intramedullary angioma as described by Clark (1954), must have operated.

Group 2.-This group consists of Cases 2 to 7 . In each instance the patient could clearly describe a hyperextension injury. Cases 2, 3, 5, and 6 followed falls on to the forehead, and in each there was local bruising. Cases 4 and 7 followed blows in the face. Particularly noteworthy is Case 6 , in which a disastrous cervical cord lesion followed a very minor accident. The mechanical basis was limitation of extension of the cervical spine due to the congenital fusion between $\mathrm{C} 2$ and 3 , and $C 6$ and 7 and $T 1$. As a result of this, in forced extension there was extreme stress of the remaining intervertebral spaces, and the anterior common ligament ruptured between the fifth and sixth cervical vertebrae. A feature of the history was the loss of speech in the early phase. This was probably due to a retropharyngeal haematoma from the torn ligaments.

Group 3.-This group contains Cases 8 to 12 . None of these patients could give a clear history of the mechanism of injury. Cases 8 and 9, however, showed radiological evidence of ossification of the anterior common ligament and so were classified as type 1 hyperextension injuries. Cases 10 and 11 had exactly similar injuries-namely, a blow over the back of the neck. It seems more likely that this would produce hyperextension than flexion of the neck. These cord injuries may therefore have been due to a massive disk prolapse in flexion or, more probably, a type 2 hyperextension injury. Follow-up radiographs in both cases showed marked degenerative changes consistent with the latter diagnosis. Case 12 was unsatisfactory in that a post-mortem examination was not permitted, and there is no evidence to show which of the mechanisms of cervical cord injury operated.

\section{Haematomyelia Due to Hyperextension Injuries}

From the foregoing, at least eight and possibly more of these 12 cases of haematomyelia were due to hyperextension injuries. These eight cases were further studied to determine the frequency of the two types of hyperextension injury. In the follow-up radiograph those cases showing bridging of the disk space by ossification of the anterior common ligament were classified as type 1 hyperextension injuries.

$$
\begin{array}{llll}
\text { Type } 1 \text { injuries } & . . & . . & 5 \text { (Cases } 3,6,7,8 \text {, and 9) } \\
\text { Type } 2 \text { injuries } & 0 & 2 \text { (Cases } 4 \text { and 5) } \\
\text { Died before follow-up } & . . & 1 \text { (Case 2) }
\end{array}
$$

The classification of Case 5 as a type 2 hyperextension injury is perhaps open to doubt, as from the original radiograph at the time of his accident there was found to be a retrospondylolisthesis of the third on the fourth cervical vertebra. This suggests a type 1 hyperextension injury with loss of the nucleus pulposus through the tear in the anterior common ligament, but the orthopaedic surgeon who originally saw the case thought these changes existed before the accident. Accordingly a type 2 hyperextension injury is more probable. Both of the cases classified as type 2 injuries thus had established degenerative changes in the cervical spine predisposing to this type of injury.

\section{Treatment of Haematomyelia Due to Hyperextension Injuries}

The primary object of treatment must be to prevent recurrence of cord compression. Two points bear on this : (1) In type 1 hyperextension injuries the displacement will recur in extension (Case 13) until reparative changes have restored stability. (2) In early cases it is impossible to distinguish with certainty the two types of hyperextension injuries. Although a type 2 hyperextension injury will not recur unless the original trauma is repeated, in view of the above it is suggested that all cases of haematomyelia following hyperextension injury should be immobilized for one month in a plaster-of-Paris collar with the neck in slight flexion. Laminectomy is contraindicated except in the presence of a spinal block on lumbar puncture or myelography. The following case shows the unavoidable hazards of cervical exploration.

Case 13.- This patient was a man aged 59. Eighteen months previous to admission, while running forward with both hands in his pockets, he tripped and struck his forehead on the ground. A transient quadriplegia lasting some ten minutes followed, with a complete recovery except that in throwing his head back to drink his daily dose of quinine he had a brief weakness or sometimes paresis of one arm. Radiographs of the cervical spine were normal. Three months after the accident a cervical laminectomy, for a diagnosis of prolapsed disk, was carried out with negative findings. After the operation he noticed stiffness and weakness of the arms and hands. He was reinvestigated some three months later and myelography was done. This showed a block in the C 6 region. Laminectomy was again undertaken, but the only finding was some scarring of the cord. After this operation the weakness and stiffness spread to the legs. When he came under our care he was having such severe burning pain in the left hand that he was unable to bear even the bedclothes on it. He was barely able to walk.

On examination a scar midline in the cervical region and gross restriction of neck movements were found. Upper limbs: Weakness and wasting of all muscles, especially intrinsics of left hand; spasticity of all groups, with increased reflexes; no reversal of supinator jerk ; left hand showed diminution of sensation in palm and radial aspect of dorsum, redness of the skin of the thenar eminence and the pulps of the fingers with extreme hyperaesthesia. Lower limbs: Weakness and spasticity all muscle groups ; increased tendon reflexes with extensor plantar responses; sensory blunting of sole and dorsum of right foot. Abdominal reflexes were absent. Sensation of trunk normal. It was considered that further recovery of the cervical cord was unlikely, and treatment was directed to muscle re-education. The pain in the left hand being temporarily abolished on three occasions by local block of the stellate ganglion, a cervical sympathectomy was carried out on the left side with relief of the causalgic pain in the left hand. No further cervical exploration was considered, as lumbar puncture showed normal pressure and no evidence of spinal block.

\section{Summary and Conclusions}

In hyperextension of the neck the spinal cord may be injured in one of two ways: (1) transient dislocation across a disk space, spontaneously reduced by muscle pull and gravity, or (2) crushing of the cord between the ligamentum flavum, which bulges forward on hyperextension, and the posterior lipping associated with preexisting spondylosis. In both injuries immediate radiographs of the cervical spine show nothing abnormal. The type 1 injury repairs itself by ossification of the anterior common ligament; this may be seen on later radiography.

All cases diagnosed as haematomyelia in a neurological clinic between 1939 and 1953 were reviewed to see how many were due to these injuries. Twelve cases were available. Of these, eight were certainly due to hyperextension injuries, two were doubtful (both blows over the back of the neck), one patient died without being able to give an adequate history and a necropsy was not carried out, and one case was non-traumatic. It would appear, therefore, that most cases of haematomyelia can be accounted for by the known mechanisms of cervical spine injury, but that there is still a small percentage in which other mechanisms operate.

In view of the difficulty of making a firm early diagnosis of which type of hyperextension injury has 
occurred, it is suggested that all cases should be immobilized in a short plaster-of-Paris collar for one month, as the type 1 injury will recur if the neck is forcibly extended (see Case 13).

Laminectomy is contraindicated unless spinal block is shown on lumbar puncture or myelography.

I am indebted to Professor F. J. Nattrass for permission to publish these cases, and to Dr. Whately Davidson for permission to reproduce the radiographs. I am grateful to Mr. J. K. Stanger for his help and advice in the preparation of this paper.

\section{REFERENCES}

Barnes, R. (1948). J Bone Jt Surg., 30B, 234

Clark, J. M. P. (1954). Ibid., 36B 418

Cramer, F., and McGowan. F. J. (1944). Surg. Gynec. Obstet., 79, 516 (1928). Proc. roy. Soc. Med., 21, 625.

Nattrass, F. J. (1944). Lancet, 2, 261.

aylor, A. R. (1951). J. Bone Jt Surg., 33B, 543.

Walshe, F. (1944), Lancet, 2,173 .

\section{PELlaGRa CAUSED BY ISONIAZID}

BY

\author{
R. J. HARRISON, M.R.C.P. \\ Physician, St. James' Hospital, London, S.W.12 \\ AND
}

M. FEIWEL, M.B., M.R.C.P.

Dermatologist, St. James' Hospital, London, S.W.12

In 1913 pellagra occurring in Britain was the subject of articles appearing in the British Medical Journal (Box, 1913 ; Mott, F. W., 1913 ; Sambon, 1913 ; Hammond, 1913); and the Lancet (Blandy, 1913). At that time Thomas (1913) suggested that certain sedative drugs such as trional and sulphonal, then in common use in mental asylums, might produce pellagra by "intoxication."

The concept of secondary pellagra, as distinct from the endemic disease, was reviewed by Bean, Spies, and Blankenhorn (1944), who listed a large number of abnormal conditions and diseases which rendered the body susceptible to this deficiency syndrome. On the other hand, pellagra was mentioned only rarely as a sequel to the use of chemical compounds.

An observation that isoniazid seemed to be of benefit in disseminated sclerosis (Kurtzke and Berlin, 1954) led to further trials of this drug.

Dr. D. B. Handley gave isoniazid in combination with pyridoxine to a number of patients with disseminated sclerosis. One of these patients, who had been existing on an inadequate diet, developed a typical pellagrous dermatitis. Three weeks after stopping the drug, while still taking the same poor diet, her symptoms persisted. There was a rapid response to nicotinamide therapy.

\section{Case History}

A spinster aged 45 had suffered from disseminated sclerosis for twenty years. Her first symptoms occurred at the age of 25 , with paraesthesiae in the legs and hands. There was a constant sensation of pins-and-needles in both legs up to the groins and in the hands and fingers up to the wrists. She also noticed that she could not appreciate hot water as acutely as before, but nevertheless never burnt herself because of this. Pin-prick, too, did not feel quite so sharp as formerly. There was no weakness of the legs, but she often felt as though she was walking on air.

After eighteen months these sensations passed off, and were later followed by pains in the legs, chiefly in the knees and calves, which were present day and night throughout the year. Then came some weakness and stiffness of the legs, especially on going upstairs. However, she could still walk long distances if she wished. On direct questioning she said that she had never had any visual or auditory symptoms, no double vision, and her speech had always been normal. There was no dyspepsia, her bowels were opened regularly, and she had never had difficulty with, or precipitancy of, micturition, or any urinary incontinence.

In 1950 she had been investigated in a neurological centre, and a diagnosis of disseminated sclerosis had been made.

History.-Her appetite had always been poor. She would have only a cup of tea and a biscuit for her breakfast, and the same again at noon. She would take a very light lunch, with little meat and fish. At tea-time she would again have tea and biscuits, and nothing further until next morning. There was no family history of fits, faints, or neurological or mental disease. Her two brothers were both well. Her father died at the age of 60 (carcinoma); her mother died aged 70 (following a stroke). At school she was reasonably intelligent, and reached the senior class. Later she worked for ten years as a bookbinder. However, for the last few years she had been doing light work in a chemist's shop, chiefly dispatching and bottling medicines. She smokes little, and drinks no alcohol.

Development of Pellagrous Dermatitis.-In May, 1955. $300 \mathrm{mg}$. of isoniazid was given daily, together with $20 \mathrm{mg}$. of pyridoxine three times a day. She took her usual inadequate meals, as her appetite did not improve. She pursued her normal activities, and did not expose herself unduly to the strong sunlight, but it was an exceptionally fine summer. During July she experienced burning sensations in the hands and feet, and the typical symmetrical pellagrous dermatitis developed on the hands and feet.

On examination her intelligence was not high; she was quite co-operative, but did not give a very good history. She was a thin woman, height $5 \mathrm{ft} .2 \mathrm{in} .(157.5 \mathrm{~cm}$.), weight 8 st. 2 lb. (51.7 kg.). Special Senses.-No anosmia. Visual acuity, good; visual fields, full to confrontation; fundi, disks normal; retinal vessels, normal; no haemorrhages or exudates. Hearing normal. Taste, normal. C.N.S.Cranial nerves, normal. Motor system: nutrition of limbsrather thin, but no wasting; no fasciculation ; slight hypotonia of arms; slightly increased tone in the legs, no clonus. Power in arms, normal ; both legs could be raised from the bed simultaneously. Other movements within normal range except for moderate weakness of dorsiflexion of the right ankle. Co-ordination.-Finger-nose test performed well, but on each side some clumsiness, especially on the left when performing repetitive movements; finger flexion normal. The impression was of slight ataxia on the left. Co-ordination of legs within normal limits. Reflexes.-Arm reflexes brisk and equal ; abdominals sluggish ; knee- and ankle-jerks brisk and equal; plantar responses extensor-the responses being obtained from the foot only. Sensations.-Pin-prick and cotton-wool were normally appreciated. Two-point discrimination, within normal limits. Postural sensibility, normal. Projection of one limb towards another with eyes closed was well performed. Vibration sense, normal. No rombergism.

Her legs were held stiffly, especially the right. Although she was a little unsteady on turning, there was no marked ataxia. The skull was normal, no bruits. Spine: no scoliosis or kyphosis; full range of movements. Heart normal in size; sounds normal, no murmurs; pulse regular; blood pressure, $105 / 75$. The respiratory system and abdomen were normal.

Skin Manifestations.-There was a typical pellagrous dermatitis, chiefly affecting the dorsal surfaces of the hands and dorsa of the feet. Around the mouth was a fine branny desquamation. The neck was not affected. The hands and feet showed a reddish-brown colour with coarse scaling, the upper boundary of the involved skin was sharply demarcated from the normal, as in the classical case (Figs. 1 and 2). The tongue was normal but angular stomatitis was marked. There was some fine scaling of the lips. Although the drugs were stopped her symptoms persisted; there was no change in her appetite or diet. 